\title{
Arendt revisited. Ein Blick auf die ersten beiden Bände der Arendt-Gesamtausgabe
}

\author{
Marti-Brander, Urs
}

DOI: https://doi.org/10.1007/s42520-020-00254-x

Posted at the Zurich Open Repository and Archive, University of Zurich ZORA URL: https://doi.org/10.5167/uzh-187864

Journal Article

Published Version

Originally published at:

Marti-Brander, Urs (2020). Arendt revisited. Ein Blick auf die ersten beiden Bände der ArendtGesamtausgabe. Neue politische Literatur, 65(2):209-227.

DOI: https://doi.org/10.1007/s42520-020-00254-x 


\title{
Arendt revisited. Ein Blick auf die ersten beiden Bände der Arendt-Gesamtausgabe
}

\author{
Urs Marti-Brander
}

(C) Gesellschaft zur wissenschaftlichen Förderung politischer Literatur e.V. and the Author(s) 2020

\section{Arendt Revisited. A Review of the First Two Volumes of Arendt's Collected Works}

\begin{abstract}
Volume 3 contains a series of short articles on Enlightenment and the Jewish question, the hidden tradition of the Jewish Pariahs, Zionism, Imperialism, the guilt question in Germany, but also Existentialism as well as essays on Franz Kafka and Stefan Zweig. A decisive issue in Arendt's thought refers to the collapse of the Western tradition of political theory in the age of Totalitarianism. It is already in the essay on Imperialism that she emphasizes the genius of Hobbes, anticipating the nihilistic ideology of the bourgeoisie. Volume 6 is dedicated to Arendt's reading of Karl Marx.
\end{abstract}

Keywords Enlightenment · Jewish question · Imperialism · Pariah · Collapse of the Western Tradition of Political Philosophy $\cdot$ Existentialism

Der Wallstein Verlag plant eine Kritische Gesamtausgabe der Werke von Hannah Arendt. Sie wird 17 Bände umfassen, von denen bislang zwei Bände, Band 3 und Band 6 erschienen sind. Zahlreiche Essays und Bücher von Arendt liegen seit Langem in unterschiedlichen Fassungen vor, viele Verlage haben einzelne Texte publiziert. Wer sich einen Gesamtüberblick über die Entwicklung ihres Denkens verschaffen will, musste bisher eigene Studien betreiben. Eine Kritische Gesamtausgabe ist seit geraumer Zeit ein Desiderat. Nun liegen zwei Bände vor; soweit abzusehen ist, werden die weiteren Bände mehr oder weniger konsequent der Chronologie folgen. Arendts erstes Buch über den Liebesbegriff von Augustin wird allerdings erst 2029

U. Marti-Brander $(\bowtie)$

Cully, Canton de Vaud, Schweiz

E-Mail: ursmarti@bluewin.ch 
erscheinen, während der erste Band zu Karl Marx die Band-Nummer 6 trägt. Ein Open-Access-Zugang wird durch die Freie Universität Berlin jeweils ein Jahr nach der Publikation bereitgestellt.

Band 3 enthält die „Sechs Essays“ von 1948, „Die verborgene Tradition. Acht Essays“ von 1976 sowie eine Reihe von Erstveröffentlichungen. Im Zentrum des Bandes stehen die politischen Fragen, die sich während und unmittelbar nach dem Zweiten Weltkrieg und der Nazi-Diktatur gestellt haben: Imperialismus, Rassismus, Nationalsozialismus, die Schuldfrage, Aufklärung, jüdische Assimilation, Pariaexistenz, Zionismus sowie die ,verborgene Tradition“. Imperialismus, Rassismus und Nationalsozialismus bewirkten den Zusammenbruch der abendländischen Tradition des politischen Denkens. Schuld daran trage ,die Existenz einer kleinen Klasse von Kapitalisten, deren Reichtum die soziale Verfassung ihrer Länder und deren Produktionskapazität die ökonomischen Systeme ihrer Völker sprengte und die daher mit gierigen Augen den Erdball absuchten nach profitablen Investierungen für überflüssiges Kapital.“ Angesichts der unendlichen Verwüstungen war dies „,wahrlich eine Bagatelle“ (S. 15). Den Zusammenhang von Imperialismus und Kapitalismus wird Arendt später in „The Origins of Totalitarianism“ genauer erläutern. Bereits im vorliegenden Artikel weist sie auf den „Mob“ hin, diese aus den „Abfällen sämtlicher Klassen“ entstandenen Schicht, die Mitschuld trägt am Zusammenbruch der abendländischen Traditionen (S. 19). Die politische Weltanschauung des Mobs wie der Bourgeoisie sei, wie Arendt glaubt, von Thomas Hobbes antizipiert worden. Hat somit Hobbes mit seiner Absage an das aristotelische politische Denken das Ende der abendländischen Tradition eingeläutet? Es geht Arendt nicht um Schuldzuweisung, die Entstehung totalitärer Herrschaft könne nicht einer bestimmten Philosophie angelastet werden, wie dies Karl Popper und andere beabsichtigt haben. Vielmehr sei es Hobbes' Verdienst, eine Umwertung der Werte diagnostiziert zu haben: Der Mensch habe keinen intrinsischen Wert mehr, sein Wert sei vom Gesetz von Angebot und Nachfrage bestimmt. Er sei demnach kein politisches Wesen mehr, die res publica sei zerstört. Als Privatmensch stehe er einer schützenden wie strafenden Staatsmacht gegenüber, die sich mit dem Versprechen rechtfertige, den Bürgerkrieg zu verhindern. Die englische Fassung weicht mehrmals von der deutschen ab, so auch im Abschnitt zu Hobbes (vgl. S. 21 ff., 259f.). Die Französische Revolution erinnere nochmals an das Ideal der res publica und des citoyen, erst nach der „Abschlachtung der Communards", der Niederschlagung der Pariser Kommune, habe die Bourgeoisie mit ihren neuen Wertvorstellungen endgültig gesiegt.

Der Artikel „Organisierte Schuld“ analysiert die perfide Propagandastrategie der Nationalsozialisten, derzufolge es keinen Unterschied gebe zwischen ihnen und den Deutschen. Die Behauptung einer Kollektivschuld des deutschen Volkes versteht Arendt vor diesem Hintergrund. Die totalitäre Politik verwischt die Unterscheidung zwischen Verbrechern und Akteuren des Widerstands, zwischen Tätern, Mitläufern und Opfern.

Ein größerer Essay fragt nach der Existenzphilosophie. Arendt hat sich nicht als Philosophin verstanden und so bleiben ihre Ausführungen zur Existenzphilosophie summarisch. Ein Thema, mit dem sie sich in späteren Jahren noch ausführlicher auseinandersetzen wird, spricht sie hier bereits an: den Gegensatz zwischen Geschichte und Philosophie. In der Nachfolge Georg Wilhelm Friedrich Hegels drohe 
die Philosophie in Spekulationen über eine mögliche Gesetzmäßigkeit des historischen Ablaufs unterzugehen. Edmund Husserls Phänomenologie bedeute dagegen eine Befreiung. Die neue philosophische Erfahrung laute nun, dass der Mensch abhängig davon ist, was er ist. Mit Friedrich Wilhelm Joseph Schelling beginne eine moderne Philosophie, die der Allgemeinheit die Einzelheit entgegensetzt. Immanuel Kant, der Philosoph der Französischen Revolution, zertrümmere den antiken Seinsbegriff und behaupte die Würde und Mündigkeit des Menschen, stelle jedoch der Autonomie die Kausalität der Natur entgegen, womit die Ideale von Freiheit und Würde aufs Neue negiert würden. Während Søren Kierkegaard von der Verlorenheit des Individuums in der Welt ausgehe, wolle Karl Marx die Einheit von Mensch und Welt wiederherstellen. Martin Heideggers Absicht bestehe darin, die Kant'sche Zertrümmerung des antiken Seinsbegriffs rückgängig zu machen. Diese Absicht führe ihn zu einem Funktionalismus, dem Arendt Hobbes' Realismus zur Seite stellt: In beiden Modellen werde der Mensch seiner Spontaneität beraubt. Es sei schließlich Karl Jaspers, der die Existenz nicht als Seinsform begreift, sondern als Form der Freiheit, worin der Mensch als Möglichkeit der Spontaneität sich gegen sein Resultatsein wendet. Der Mensch werde als Wesen bestimmt, das mehr ist als sein Selbst und mehr will als sich selbst. Damit trete die Existenzphilosophie aus der Periode ihrer „Selbstischkeit“ heraus. Arendts Durchgang durch die nachhegelsche Philosophie bleibt oberflächlich. So ist der Gegensatz zwischen Hegel und Marx nicht jener von Geist und Materie, sondern von Mystifikation und Rationalität, und gewiss war es nicht Marx' Absicht, Existenzphilosophie zu betreiben. Arendt hat sich später von diesem Aufsatz distanziert und falsche Interpretationen eingeräumt (S. 357).

„Die verborgene Tradition“ ist ein Essay über jüdische Literatur und die ,Figur' des Paria. Die Juden, die sich in Europa nicht assimilieren wollten, sondern versucht hatten, ,die frohe Botschaft der Emanzipation so ernst zu nehmen, wie sie nie gemeint gewesen war, und als Juden Menschen zu sein", befreiten sich als Individuen in Opposition zu ihrer jüdischen wie nicht-jüdischen Umgebung selbst. Das Schicksal des jüdischen Volks in Europa war das eines Paria-Volks; dies sei jenen am klarsten zu Bewusstsein gekommen, welche die zweideutige Freiheit der Emanzipation und die zweideutige Gleichheit der Assimilation erfahren hatten. In ihrer Existenz als Parias außerhalb der Gesellschaft „spiegelte sich das politische Dasein des Volkes als Ganzes wider" (S. 65). Sie konnten die Figur des Parias konzipieren, ,die eine für die moderne Menschheit sehr bedeutsame neue Idee vom Menschen enthält.“ Ihr Einfluss auf die nicht-jüdische Welt stand ,in groteskem Widerspruch" zu ihrer Wirkungslosigkeit im eignen Volk. Arendt illustriert dies an den Beispielen von Heinrich Heine, Bernard Lazare, Charlie Chaplin und Franz Kafka. Der Paria stehe außerhalb der Rangordnungen der Gesellschaft und verweigere sich ihr, in seiner unbekümmerten, heidnischen Frechheit sei er volkstümlich. Heine erkennt in seinem Gedicht „Apollogott“ im Spieler, Freigeist und Komödianten Rabbi Faibusch Phoibos Apollo wieder. Der Paria ist zugleich Schlemihl, der seinen Schatten verkauft. Er ist sich der Inkongruenz zwischen der Schöpfung, vor deren Erhabenheit alles gleich ist, und den gesellschaftlich fabrizierten Rangunterschieden bewusst. Die Rollen kehren sich um, nicht mehr der Verachtete ist Schlemihl, sondern jene, welche die Güter der Natur eintauschen gegen die Götzen des gesell- 
schaftlichen Vorteils. So erfuhr Heine an „der natürlichen Distanz des Paria zu allem Menschenwerk" das Wesen der Freiheit. Sein Zorn gilt den Tyrannen wie dem Volk, das sie erträgt. Bernard Lazare, der Initiator der Dreyfus-Bewegung, repräsentiert für Arendt den Typus des politisch bewussten Paria. Mit den Begriffen ,Parvenü ‘ und ,Paria' sind zwei Möglichkeiten angesprochen, die den europäischen Juden im letzten Jahrhundert offen standen: Assimilation mit dem Ziel, gesellschaftlich aufzusteigen und die Abstammung zu verleugnen, oder Bejahung der Herkunft, die Bereitschaft, außerhalb der sozialen Rangunterschiede zu leben. Der bewusste Paria akzeptiere die Ungerechtigkeit nicht. Er werde ,zum Vertreter eines unterdrückten Volkes, das seinen Freiheitskampf in Verbindung mit den nationalen und sozialen Freiheitskämpfen aller Unterdrückten Europas führt“ (S. 71). Lazare kritisiert die Ideologie der Assimilation. Verlange man von den Juden, ihre Charaktereigenschaften aufzugeben und sich nur noch durch äußerliche Eigenschaften auszuzeichnen, werde man sie erst recht diskriminieren. Lazare entdeckte eine doppelte Knechtschaft: sowohl die nicht-jüdische Umgebung als auch die vermögenden jüdischen Parvenüs seien den Parias feindlich gesinnt. Der bewusste Paria müsse zum Rebellen werden, weil er sonst mitverantwortlich sei für die Schändung der Menschheit in ihm. Der Gegensatz von ,Parvenü “ und ,Paria ‘ wird Arendts politisches Denken fortan in unterschiedlichen Formen leiten: Wohlstand und Freiheit, bourgeois und citoyen, Gesellschaft und Politik, Kapitalakkumulation und soziale Gerechtigkeit.

Charlie Chaplin steht bei Arendt für den ohnmächtigen kleinen Mann aus dem Volk, der mit der Gesellschaft, ihrer Ordnung und ihren Gesetzen permanent in Konflikt gerät. Er ist grundsätzlich suspekt, aber zugleich unschuldig, weil der Zusammenhang von Vergehen und Strafe unverständlich und jede Art der Bestrafung willkürlich ist. Franz Kafka erlebt den Konflikt zwischen Gesellschaft und PariaExistenz auf seine besondere Weise. Für ihn stellt sich die Frage, ob dem Außenseiter überhaupt noch Realität zukommt, oder ob er ein Niemand ist, wohlwissend, dass die Gesellschaft aus lauter Niemanden besteht. Im Roman „Das Schloss“ sieht Arendt die Problematik der Assimilation beschrieben. K. hofft vergeblich, ununterscheidbar zu werden. Er will nichts anderes als sein Menschenrecht. In der Hoffnung auf Assimilation tut er, was von Juden erwartet wird, er vereinzelt sich, muss aber erfahren, dass die Menschenrechte für ihn nicht existieren. Die Freiheit des Parias ist sinnlos, weil der Wille, etwas in der Welt zu bewegen, nicht vorgesehen ist. Ausführlicher präsentiert Arendt Kafkas Werk in „Franz Kafka, von neuem gewürdigt“ (deutsche und englische Version). In diesem Kontext versteht sie den Niedergang des citoyen und das Ende der Französischen Revolution - den Versuch, die Welt nach menschlichen Gesetzen zu regieren - als Basis des klassischen Romans.

„Die verborgene Tradition“ ist eine faszinierende, leidenschaftliche Schrift, die Fragen aufwirft. Die Aufwertung der Paria-Existenz geht mit der Abwertung der Parvenü-Existenz einher, einem Großteil der europäischen Juden wird unterstellt, den falschen Weg gewählt zu haben. Überdies ist nicht klar, wie die Paria-ParvenüTheorie aufgenommen und ob sie überhaupt verstanden worden ist. So schreibt Hans Blumenberg in einer Rezension:

Wie war es möglich, dass in der vergangenen Epoche nicht der gemeingefährliche Gewohnheitsverbrecher, der asoziale Außenseiter in der Organisation des 
Mordes eingespannt und zum Vollstrecker ihrer Aktionen wurde, sondern der „,biedere“"Bürger, der „Familienvater“? Ahrendt [sic] entwickelt ihre Erklärung für dieses unfassbare Paradox aus der Erscheinung des Paria, die in einem totalitären System gerade in dem Spießbürger und dem Familienvater ihre moderne Auferstehung erlebt. Gerade ihn treibt die beständige Bedrohung der elementaren Existenz und des nackten Daseins [...] zum verzweifelten Gehorsam, zur moralischen Bedingungslosigkeit und zur Missachtung der Schuld, sie treibt ihn in jene Situation des Paria, die nur die Wahl hat, dienlicher Gegenstand oder überhaupt nicht zu sein. (S. 450)

Blumenberg hat offensichtlich Arendts Argument nicht verstanden. Er verwechselt den Paria mit dem Mob, der im Verbund mit der Bourgeoisie der Festigung der totalitären Ordnung zuarbeitet.

„Juden in der Welt von gestern“ ist schließlich eine Besprechung von Stefan Zweigs Autobiografie. Zweig zeichnet darin auf, wie sich nach 1914 die Welt immer unheimlicher verändert hat, wie er aus dem „Paradies des gebildeten Genusses“ vertrieben wurde und der Schimpf der Demütigung das Glück des Ruhms verdrängt hat. Arendt kritisiert in ihrer Besprechung die naive, unpolitische Haltung der jüdischen Intellektuellen Wiens (vgl. die englische Version, S. 165-173).

„Die verborgene Tradition“ ist ein Kapitel in den „Sechs Essays“, zugleich werden in Band 3 der Gesamtausgabe unter diesem Titel zwei Artikel versammelt. Der Artikel „Aufklärung und Judenfrage“ geht von der Feststellung aus, dass die Aufklärung, verstanden als die nicht-jüdische europäische Welt, nach diesem Verhältnis gefragt habe. Gotthold Ephraim Lessing gründete seine Argumente auf der Annahme einer allen Menschen gemeinsamen Vernunft. Dies führt zur Forderung der Toleranz, die Wahrheit wird zum vernachlässigbaren Wert, erst die Suche nach ihr mache den Wert des Menschen aus. Arendt erklärt anhand der Emanzipationsdebatten des ausgehenden 18. Jahrhunderts, mit welchen Argumenten für die Assimilation der Juden plädiert wurde, und wieso sie letztlich doch wieder als Fremde wahrgenommen wurden. Geschichte als das Suchen nach der Wahrheit liege für Lessing nicht in der Vergangenheit, sondern beginne mit der Aufklärung, der Mündigkeit der Menschen. Mendelssohn verabsolutiere die Vernunft; das autonome, selbstdenkende Individuum vertraut auf sie allein und erhofft die intellektuelle Vervollkommnung der Menschheit, die Rückverankerung in der Geschichte ist unnötig. Die Vernunft sei für Moses Mendelssohn identisch mit der jüdischen Religion. Christian Wilhelm Dohm, Autor von „Über die bürgerliche Verbesserung der Juden“, identifiziere die Geschichte der Juden mit der Geschichte ihrer Minderwertigkeit. Es ist die Geschichte einer schlechten Vergangenheit, von der sich die Juden befreien müssen, um eingebürgert werden zu können. Johann Gottfried Herder bereite mit seiner Kritik an der Aufklärung die Romantik vor. Die Wiederentdeckung der Geschichte gehe mit der erneuten Entdeckung der Verschiedenheit von Menschen und Völkern einher. Die Juden blieben für Europa ein fremdes, asiatisches Volk. Dennoch strebt Herder ihre Assimilation an. Dies setze Bildung voraus, und damit in Arendts Urteil die Negation, den Verlust der Geschichte der Juden.

„Der Zionismus aus heutiger Sicht“, in englischer Sprache 1945 erschienen, auf Deutsch 1948, setzt sich kritisch mit der zionistischen Bewegung auseinan- 
der. Arendt war bis 1943 Mitglied der World Zionist Organization, distanzierte sich jedoch später vom Zionismus und plädierte für eine jüdisch-palästinensische Konföderation. Die Schrift scheint angesichts der gegenwärtigen Situation nichts von ihrer Aktualität eingebüßt zu haben, im Gegenteil. 1944 forderte die zionistische Weltorganisation ein freies und demokratisches jüdisches Gemeinwesen, das ganz Palästina umfassen sollte. Die arabischen Bürger fanden keine Erwähnung, ,so dass ihnen offenkundig nichts anderes bleibt, als zwischen freiwilliger Emigration und einer Existenz als Bürger zweiter Klasse zu wählen“ (S. 130). Die Zielvorstellungen der Zionisten hatten sich durchgesetzt, die nationalistischen Parteien verfolgten eine noch härtere Politik und forderten die Umsiedlung der arabischen Bevölkerung. Ein Wille zur Verständigung zeichnete sich nicht ab. Käme es zu kriegerischen Auseinandersetzungen, wären die Juden auf die Unterstützung einer Großmacht angewiesen, welche die Interessen der Anwohner nicht ignorieren könne; eine solche Konstellation würde überdies einem neuen Antisemitismus Vorschub leisten, wie Arendt befürchtet. Die Opposition zwischen Zionismus und Revisionismus erklärt sich aus unterschiedlichen politischen Zielen: Sozialismus und Nationalismus. Der sozialistische Zionismus gab sich laut Arendt der Illusion hin, in einem umkämpften Territorium die gute Ordnung zu realisieren und zur Weltrevolution beizutragen. Politisch hätten die Zionisten nichts bewirkt, ihre revolutionären Energien seien verpufft, ihre chauvinistischen Forderungen richteten sich nicht gegen die Feinde des jüdischen Volks, sondern gegen seine potenziellen Freunde und Nachbarn. Arendt ist in ihrer Kritik an den falschen oder fehlenden politischen Konzeptionen der zionistischen und revisionistischen Parteien unerbittlich. Theodor Herzls Ansicht, die Antisemiten seien die verlässlichsten Verbündeten der Juden, die Geschäfte der zionistischen Organisation mit den Nationalsozialisten, die fehlende Kritik an der politischen Rolle des jüdischen Finanzkapitals im Ausland, Herzls Verhandlungen mit der türkischen Regierung während des Genozids an den Armeniern, die Unterwerfung unter die politischen Pläne der Großmächte, das fehlende Verständnis für die Bedrohungen durch den Imperialismus, der fehlende Mut der linken Zionisten, der Kampf jüdischer Gewerkschaften gegen arabische Arbeiter, die Geringschätzung der Volkssouveränität - das Register ist lang. Nur gelegentlich verweist Arendt auf die schwierigen Bedingungen, unter denen die palästinensischen Juden überhaupt politisch handlungsfähig waren. Ihr zufolge hätte die von Bernard Lazare vorgezeichnete Alternative darin bestanden, im Verbund mit allen fortschrittlichen Kräften Europas eine revolutionäre Bewegung zu bilden. Eine Politik jedoch, die auf die Protektion einer imperialen Macht baue und sich den guten Willen der Nachbarn verscherze, könne nur auf Torheit beruhen, so Arendts Konklusion.

Der umfangreiche Band 6 trägt den Titel „,The Modern Challenge to Tradition: Fragmente eines Buchs“. Er umfasst drei Teile, betitelt mit: „The Great Tradition“ (1952/1953), ,The Modern Challenge to Tradition“ (1953) und „Eine Art Buch A Book that can't be written“ (1954). Ein großer Teil der Fragmente ist Karl Marx gewidmet. Worum genau es bei dieser Auseinandersetzung mit Marx geht, ist nicht leicht zu beantworten. Arendt schreibt als Philosophin, und sie schreibt über Marx als Philosophen. Die Kritik des jungen Marx am Absolutismus sowie des späteren Marx am Kapitalismus steht dabei nicht im Vordergrund. Das Interesse an Marx erklärt sich vielmehr aus dem Umstand, dass er, allenfalls mit Søren Kierkegaard und 
Friedrich Nietzsche, die Tradition der abendländischen Philosophie beschließt. Im ersten Teil ihrer Auseinandersetzung mit Marx geht es um Ideologie, Terror und totalitäre Herrschaft. Die totalitäre Ideologie behandele Menschen als Material, woran übermenschliche Gesetze von Natur und Geschichte vollzogen würden. Den nationalsozialistischen Rassegesetzen liege Charles Darwins Vorstellung vom Menschen als eines zufälligen Resultats einer Naturentwicklung zugrunde, dem Glauben der Bolschewisten an Geschichtsgesetze liege Marx' Vorstellung von der menschlichen Gesellschaft als dem Resultat eines gigantischen Geschichtsprozesses zugrunde, der seinem Ende entgegenrast und sich selbst als Geschichte aus der Welt schafft (S. 13, 31, 73). Darwins Kampf der Rassen und Marx' Kampf der Klassen gründen auf derselben Logik, wie Arendt glaubt - eine fragwürdige Ansicht. Will Arendt sagen, die Evolutionstheorie von Darwin und die Modernisierungstheorie von Marx seien wissenschaftlich falsch? Marx schreibt:

An und für sich handelt es sich nicht um den höheren oder niedrigeren Entwicklungsgrad der gesellschaftlichen Antagonismen, welche aus den Naturgesetzen der kapitalistischen Produktion entspringen. Es handelt sich um diese Gesetze selbst, um diese mit eherner Notwendigkeit wirkenden und sich durchsetzenden Tendenzen. Das industriell entwickeltere Land zeigt dem minder entwickelten nur das Bild der eignen Zukunft. (MEW, Bd. 23, S. 11)

Die Geschichte hat dies bislang bestätigt. Arendt hält die Gesetze der Geschichte, die Natur- und Sozialwissenschaften generell für bloße Ideologien, ihr eigenes Wissen, ihre Gewissheiten resultieren aus einer unmittelbaren Erfahrung. Wie steht es mit Kants bekanntem Aufsatz „Idee zu einer allgemeinen Geschichte in weltbürgerlicher Absicht"? Er wollte die menschliche Geschichte naturgesetzlich erklären und prüfen, ob nicht im ,widersinnigen Gange menschlicher Dinge“ eine Naturabsicht entdeckt werden kann, niemand wird ihm deshalb totalitäre Absichten unterstellen. Später wird Arendt festhalten, die Schuld an der Entstehung totalitärer Herrschaft sowie am Aufkommen des Antisemitismus sei nicht einer Ideologie zuzuweisen, sondern „unterhalb der Oberfläche der europäischen Geschichte“ zu suchen. Dies wird nicht weiter erläutert; Arendt bemerkt lediglich, diese „Unterschicht“ der europäischen Geschichte sei „dem Licht der Öffentlichkeit und der Aufmerksamkeit der Aufgeklärten entzogen“.. Dass die Arbeiterklasse die eigentliche Substanz der Ideologie selbst sei (S. 19f), ist wiederum eine schwer verständliche Behauptung. Die Herausbildung einer Klasse der Lohnarbeiter im Zuge der Industrialisierung ist eine unbestreitbare Entwicklung; die Erfahrung, dass Klassen im historischen Verlauf untergehen und neue Klassen sich bilden, kann zur Legitimation einer mörderischen Politik missbraucht werden, ist aber nichtsdestoweniger eine historische Realität (S. 42). Wie Marx betont: Die Existenz von sozialen Klassen mit gegensätzlichen Interessen ist von ihm nicht erfunden, vielmehr von liberalen Autoren entdeckt worden. Arendt beschäftigt etwas anderes. Bereits in der Darlegung der Philosophie von Hobbes hält sie fest:

\footnotetext{
${ }^{1}$ Arendt, Hannah: Elemente und Ursprünge totaler Herrschaft, EVA, Frankfurt a. M. 1955 (engl. 1951), S. $22 \mathrm{f}$.
} 
The same insight into the necessary instability of a community founded on power is philosophically expressed in the concept of the endless process of history. Corresponding to the constant necessity of increasing the power of the state, this process must take the form of progress - a progress that inexorably catches up individuals, peoples, and finally all humanity, regardless of human welfare. (Bd. 3, S. 260)

Die Machtakkumulation müsse schließlich, so mutmaßt Arendt, im von den Liberalen herbeigewünschten Weltstaat enden, in einem absoluten und erdumspannenden Machtmonopol. Der Fortschritt als solcher steht mithin unter Anklage, der Liberalismus als Ideologie des Fortschrittsdenkens. Handelt es sich dabei nicht um einen Kampf gegen Windmühlen? Oder ist zu unterscheiden zwischen dem endlosen Prozess der Geschichte und jenem der unaufhaltsamen Akkumulation von Kapital und staatlicher Macht? ,[...] the individual is invited [...] to become a part of a blind power-machine which sets no limit to his accumulation of capital, no bounds to his career as a self-made man“ (Bd. 3, S. 261). Arendt betont mehrmals Marx' intellektuelle Größe und verneint konsequent seine angebliche Schuld am Totalitarismus. ,,[...] so verschieden diese beiden Ideologien voneinander sind, so großartig erfüllt mit den besten abendländischen Traditionen der dialektische Materialismus, so kläglich vulgär, wiewohl auf einem echten Erfahrungselement basierend der Rassismus, gemeinsam ist ihnen der Glaube an ein unerbittliches Bewegungsgesetz" (S. 31). Andernorts betont Arendt, die Linie von Aristoteles zu Marx sei ungleich bedeutsamer als jene von Marx zu Stalin (S. 247).

Arendt diagnostiziert eine Krise der westlichen Politik und des westlichen politischen Denkens. Was die von wirtschaftsliberalen Doktrinen inspirierten Umwälzungen der Sozialordnungen bewirkt haben - rücksichtslose Konkurrenz und Atomisierung der Gesellschaft sind die meist genannten Stichworte - haben frühere Autoren, von Jean-Jacques Rousseau über Adam Ferguson und Marx bis zu Nietzsche, bereits beobachtet. Arendt setzt diese Tradition fort, kritisiert aber den Umstand, dass das politische Denken fortan von den Sozialwissenschaften beherrscht werde. Montesquieus Frage nach den Prinzipien der Staatsform (Tugend, Ehrgeiz, Habgier, Luxus, Mäßigung, Gesetz, Furcht) werde nicht mehr gestellt (S. 11f). Soziologie und Psychologie haben ihren Ursprung laut Arendt in einem Liberalismus, der Politik als Zusammenspiel von Gesellschaft und Individuum versteht. Menschen werden geprägt von der Gesellschaft; zwar haben sie eine Seele, allerdings nur, wenn sie mehr sind als bloße Teile der Gesellschaft. In der Gesellschaft sorgen Gewohnheiten und Konventionen, Sitten und öffentliche Moral für ihr Wohlverhalten. Das moderne Individuum hat eine Gesellschaft überlebt, die nicht mehr existiert. Dies bestätigen die Sozialwissenschaften, sie übertragen individuelle Verhaltensmuster auf die Gesellschaft, in der solche Verhaltensweisen gar nicht vorkommen. Arendt zitiert Platons Bemerkung, Verfassungen entstammten den Sitten der Bürger (Plato: Republic, Book VIII, 544d, S. 70). Sie ruft die Unterscheidung von res publica und res privata in Erinnerung. Politische Organisationsformen betreffen Angelegenheiten, die alle Bürger angehen. Dagegen gehorche die totalitäre Regierung ausschließlich den übermenschlichen Gesetzen der Natur und der Geschichte. Glaubt sie, dass 
solche Gesetze tatsächlich existieren, oder will sie nicht eher sagen, dass totalitäre Regierungen deren Existenz behaupten, um ihre Politik zu legitimieren?

Arendts Argumentationsweise beruht häufig auf Impressionen, sie urteilt über den Sinn und die Absichten von Wissenschaften, ohne den geringsten Beleg für ihre Behauptungen zu liefern (S. 68-72). Es sind Menschen, die Geschichte machen, allenfalls Gesetze des zivilisatorischen Fort- oder Rückschritts entdecken oder zu entdecken glauben. „Die Menschengeschichte unterscheidet sich dadurch von der Naturgeschichte, dass wir die eine gemacht und die andere nicht gemacht haben“, so schreibt Marx im Anschluss an Giambattista Vico. Es handelt sich hierbei nicht um übernatürliche Gesetze, sondern um die Bildungsgeschichte, die Erfindungsgabe des Menschen (MEW, Bd. 23, S. 392f.). Die Menschen machen ihre eigene Geschichte, aber sie machen sie nicht aus freien Stücken, nicht unter selbstgewählten Umständen (MEW, Bd. 8, S. 115). Marx attestiert den Menschen zumindest ein wenig Handlungs- und Gestaltungsfreiheit. Freilich kannte er die besonderen Bedingungen totaler Herrschaft nicht. Arendts wissenschaftsfeindliche Haltung kommt im folgenden Zitat zum Ausdruck: „The tremendous intellectual change which took place in the middle of the last century consisted in the refusal to view or accept

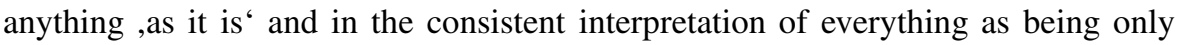
a stage of some further development" (S. 73). Der Fortschritt ist eine zweideutige Sache, daran zu erinnern ist trivial. Dass sich die Welt aufgrund politischer und industrieller Revolutionen seit dem ausgehenden 18. Jahrhundert verändert hat und immer spürbarer verändert, konnte Arendt nicht ignorieren. Dass sie diese Entwicklung mit Sorge betrachtete, ist verständlich, weniger allerdings, dass sie die reale Erfahrung umdeutete in eine bloße Legitimationsideologie.

Der Vortrag „Von Hegel zu Marx“ wird in zwei Varianten abgedruckt. Er nimmt das Thema einer Tradition der abendländischen Philosophie auf, einer Tradition, die mit Marx und Nietzsche an ihr Ende gelangt sei. Die beiden Denker stünden für eine „Umstülpung“ Hegels (Marx) und eine Umkehrung Platons (Nietzsche). Beide übernähmen den Rahmen der Tradition, leugneten aber deren Autorität. Werde die Tradition ohne religiös fundierte Autorität übernommen, gehe der in Form der Autorität ausgesprochene Anspruch an den Menschen verloren. Marx habe Hegels Dialektik ihres substanziellen Gehalts beraubt und damit das „Prozessdenken“ ermöglicht, das ,,in der vernichtenden Logik totalitärer Herrschaftsapparate“ geendet habe (S. 90). Die weitere Darlegung ist überraschend:

Marx bricht „mit allen überkommenen Wertungen der politischen Philosophie, denen zufolge Denken höher steht als Handeln und Politik nur dazu da ist, den bios theoretikos, das kontemplative Leben der Philosophen oder die von der Welt abgekehrte, christliche Betrachtung Gottes zu ermöglichen und zu sichern" (S. 91). Nun ist zwar richtig, dass Aristoteles in der "Nikomachischen Ethik“ die theoretische Lebensweise über die praktische stellt, doch sein Argument ist differenzierter: Das höchste Glück finde der Mensch in der Philosophie, im reinen, zweckfreien Betrachten. Da es in Muße bestehe, erwiesen sich die praktischen Tugenden als unvollkommen, sei doch Politik mit Muße unvereinbar und nicht Selbstzweck. Die politische Gemeinschaft aber sei Mittel zum Zweck der Realisierung des höchsten Guts. Der vollkommene Staat sei um des Lebens willen entstanden und bestehe um des vollkommenen Lebens willen. Er existiere von Natur aus und verkörpere 
das höchste Ziel, wonach jeder Verbund strebe. Natur - physis - meint die Vollendung eines Prozesses. Der Mensch sei von Natur aus ein politisches Wesen, das nur im Staat zu verwirklichen vermag, was in ihm angelegt ist (Aristoteles: Pol. I 2, 1252b-1253b). Eine politische Philosophie, die das Denken über das Handeln stellt, sich von der Welt abkehrt und der Gottesbetrachtung zuwendet, wäre eben keine politische Philosophie mehr. Arendts Argumentation wird weder Aristoteles noch Marx gerecht. Aristoteles wie Marx argumentieren perfektionistisch. Für Aristoteles geht der Staat dem Einzelnen voraus. Zwar können Menschen außerhalb der politischen Gemeinschaft überleben, doch nur in der Gemeinschaft können sie ihre guten Anlagen entfalten. Ganz ähnlich formuliert Marx, die künftige Gemeinschaft sei eine Assoziation, in der die freie Entwicklung eines jeden die Bedingung für die freie Entwicklung aller sei (MEW, Bd. 4, S. 482). Einen weiteren Traditionsbruch sieht Arendt in der Aufwertung der Arbeit als hervorragende menschliche Tätigkeit und kreative Macht. Immerhin sieht sie Marx' Größe darin, die Arbeit ins Zentrum seiner Lehre gestellt zu haben, während die traditionelle politische Philosophie davon die Augen abgewandt habe. Die politische Frage nach der Notwendigkeit der Arbeit im menschlichen Leben und ihrer alles beherrschenden Rolle in der modernen Welt ist damit aber nicht beantwortet, wie Arendt festhält - ohne zu erläutern, wie die Frage und eine mögliche Antwort lauten könnten (S. 99f). Dass der Mensch gezwungen ist, zu arbeiten um zu überleben, mithin nicht frei ist, es nicht zu tun, ist doch wohl unbestritten. Marx' Absicht war es, zu zeigen, dass die Unfreiheit des Menschen, der gezwungen ist, für einen anderen zu arbeiten, nicht legitimiert werden kann, dass umgekehrt die selbstbestimmte Arbeit zur Entwicklung der freien Individualität des Menschen beiträgt (MEW, Bd. 23, S. 789). Wie ist die zweideutige Marx-Kritik zu verstehen? Wenn laut Aristoteles der Mensch von Natur aus ein politisches Wesen ist, trifft dies auf den Sklaven nicht zu: Wer mit dem Körper arbeiten kann, ist von Natur aus Sklave (Aristoteles: Pol. I 2, 1252a). Das politisch ,Richtige' ist in der Ordnung der Natur vorgegeben, sie weist jedem Wesen seinen Platz zu. Von dieser aristotelischen Auffassung lässt Arendt sich inspirieren, ohne sie zu rechtfertigen. Marx' Glorifizierung der Arbeit habe keinen der Gründe beseitigt, welche die Tradition anzuführen wusste, wenn sie dem nur arbeitenden Menschen volle menschliche Freiheit und Gleichberechtigung aberkannte. Was ist gemeint mit den „Gründen“, sind es ,gute 'Gründe? Folgt daraus, dass nur müßigen Menschen Freiheit und Gleichberechtigung zusteht? Tripalium, das Wort, aus dem sich mehrere Begriffe für Arbeit herleiten, bezeichnet ein Folterinstrument, mit dem Sklaven bestraft wurden. Laut Aristoteles ist ein Mensch, der arbeiten muss, um zu überleben - ob es sich um einen Sklaven oder freien Bürger handelt - nicht frei, weil ihn die Not oder ein Herr zur Arbeit zwingt. Arendt meint, Marx sehe die einzig legitime Befriedigung eines Interesses in der Arbeit. Doch Marx sieht das Interesse des Arbeiters in der Selbsttätigkeit und Autonomie, in der Freiheit, arbeiten zu können, ohne einem Herrn untertan zu sein, ja sogar darin, in der Arbeit Erfüllung zu finden. Arendt verdrängt diese Idee, sie passt nicht in ihr Denkschema. Ohnehin rezipiert sie Marx höchst selektiv. Arbeit könne nie produktiv, der arbeitende Mensch nie frei sein. Menschen müssten arbeiten, um zu überleben, die Organisation der Arbeit erfordere Zwang in Form der Versklavung oder zumindest von prekären Arbeitsund Lebensbedingungen, kurz: Arbeit erfordere eine Aufspaltung der Menschheit in 
Herrschende und Dienende. All dies ist seit Aristoteles bekannt und hat immer wieder der Absicht gedient, den Müßiggang der Wohlhabenden und das harte Los der Arbeitenden als naturgewollt zu legitimieren. Erstaunlich ist, wie unkritisch Arendt dieser aristotelischen Naturwissenschaft vertraut. Dies heißt nicht, dass sie die Institution der Sklaverei rechtfertigt, die „Gewalt, mit der ein Teil der Menschheit sich auf Kosten eines Anderen“ vom Zwang zur Arbeit befreit, wohl aber fordert sie die Grenzziehung zwischen den Bereichen von Freiheit und Arbeit:

Die Fähigkeit, in einer Welt zu leben, kann sich nur in dem Maße realisieren, als Menschen gewillt sind, die Lebensprozesse zu transzendieren und sich ihnen zu entfremden, während umgekehrt die Vitalität und Lebendigkeit menschlichen Lebens nur in dem Maße gewahrt werden können, als Menschen bereit sind, die Last, die Mühe und Arbeit des Lebens auf sich zu nehmen. ${ }^{2}$

Wie kann man sich den Lebensprozessen entfremden? Ist nicht eher Hannah Arendts Anthropologie befremdlich? Menschen sollen in zwei strikt voneinander getrennten Welten leben, sich in der Arbeit von aller Politik fernhalten, in der Politik alles Lebenswichtige verdrängen und vergessen. Es erübrigt sich hier, auf die zahlreichen kritischen Debatten hinzuweisen, in denen Arendts radikale Opposition von sozialen und politischen Bereichen analysiert wird.

Mehrere Notizen, Entwürfe und Texte setzen sich mit der totalitären Herrschaft auseinander. Unter dem Titel „Understanding and Politics“ sind vier Entwürfe und Artikel versammelt. Arendt widerspricht der verbreiteten, wenn auch nach wie vor von den Humanwissenschaften anerkannten Auffassung, um den Totalitarismus zu verstehen und zu bekämpfen, brauche es die vereinten wissenschaftlichen Anstrengungen historischer, ökonomischer, soziologischer und psychologischer Forschung. Verstehen benötigt keine wissenschaftliche Hilfe. Die Natur des Totalitarismus gilt es zu verstehen. Er ist die radikalste Verneinung von Freiheit. Niemand wird dem widersprechen wollen, doch sollte daraus etwa folgen, dass Wissenschaft überflüssig ist? Arendt hält zunächst fest, dass in Europa die gesamte Struktur von Moral, Geboten und Verboten zerstört worden sei. Dieser Zusammenbruch werde von vielen Menschen nicht verstanden, die glaubten, die alte Ordnung könne restauriert werden. Montesquieu ist für Arendt der maßgebliche politische Denker. Ihm zufolge werden sich in einem Prozess der Korruption und des Niedergangs die Sitten länger halten als die Gesetze. Die meisten europäischen Völker sind noch von den Sitten geleitet, wird jedoch der Despotismus sich dereinst durchsetzen, werden diese nichts mehr ausrichten (S. 135, der Nachweis ist von Arendt falsch zitiert, es handelt sich um Buch VIII, Kap. 8). Zu verstehen gilt es letztlich nur, was von Beginn an bekannt war: Totale Herrschaft leugnet menschliche Freiheit radikal. Arendt widerspricht sich häufig: Um die Natur totaler Herrschaft zu verstehen, müssten ihre Ursprünge und ihre Struktur analysiert und die traditionellen Fragen bezüglich der Natur und der Prinzipien totaler Herrschaft gestellt werden. Dagegen sei jede Wissenschaft falsch, die nach Ursachen und Konsequenzen frage - was gilt nun? Die Polemik gegen die Wissenschaften und ihre angeblich engen Grenzen schierer Faktizität sind für das Verständnis nicht hilfreich, die Berufung auf eine Tradition der politischen

\footnotetext{
2 Dies.: Vita activa oder Vom tätigen Leben, Piper, München/Zürich 1981 (engl. 1958), S. 109.
} 
Philosophie, die - einmal mit Kant, einmal mit Marx - ihr Ende findet, ebenso wenig.

Marx war, so Arendt in „Religion and Politics“, überzeugt, politisches Handeln gehe stets mit Gewalt einher. Wiederum unterstellt sie eine philosophische Überzeugung, ohne sich um historische Fakten zu kümmern. Marx war 1848 und 1871 Zeuge der brutalen Unterdrückung der Arbeiter in Frankreich, er hat die mit der gewaltsamen Kolonisation außereuropäischer Regionen einhergehende kapitalistische Akkumulation studiert und akribisch beschrieben. Marx verstand, so Arendt, den historischen Akteur als Homo faber, Gewalt ist somit bereits im Spiel, wenn es um die Produktion von Gütern geht, jede Art der Fabrikation tut der Materie Gewalt an. Mit dieser Ansicht stimmt Marx laut Arendt mit der ganzen Tradition des politischen Denkens überein. Sie erwähnt mehrmals drei Axiome von Marx' Denken: Arbeit ist der Schöpfer des Menschen, Gewalt ist der Geburtshelfer der Geschichte, niemand, der andere beherrscht, kann frei sein (S. 275, 289). Eine andere Version des dritten Axioms lautet: Die Philosophen haben die Welt nur verschieden interpretiert, es kommt darauf an, sie zu verändern (S. 393). Es ist laut Arendt nicht ein revolutionäres Temperament, das Marx zu diesen Einsichten geführt hat, sondern eine humanistische Gesinnung, die den Menschen als handelndes und Geschichte machendes Wesen versteht. Anders als Kant und Hegel vertraue Marx keiner List der Natur oder der Vernunft. Vielmehr erhebe er den Arbeiter zum politischen Akteur; wer Güter produzieren kann, kann ebenso die Geschichte gestalten (S. 197f). Wie alle ernsthaften Philosophen seit der Französischen Revolution ist Marx mit dem Rätsel konfrontiert, dass Handeln im Gegensatz zur Produktion kaum je seinen Zweck realisieren wird. Handlungen finden statt in einem Geflecht von widerstreitenden Absichten und Kräften, dennoch ist die Geschichte sinnvoll und für die Menschen verständlich (S. 217).

Was verbindet Marx mit der Tradition des politischen Denkens? Der Artikel „Karl Marx and the Tradition of Political Thought“ gibt darüber Aufschluss. In Arendts Darlegung überlagern sich mehrere Argumentationslinien, der Zusammenhang des Ganzen ist nicht immer ersichtlich: „It has never been easy to think and write about KM“ (S. 245). In der Tat. Die ,Erfindung ' des Klassenkampfs war nicht Marx' Verdienst, wie er selbst betont hat. Klassenkonflikte gab es bereits im antiken Griechenland. Die Bedeutung von Marx' Werk liegt laut Arendt in seiner Auseinandersetzung mit den Problemen der Arbeit und der Geschichte. Er habe entdeckt, dass Arbeit in der modernen Welt einen entscheidenden Wandel erlebt hat und fortan alle menschlichen Aktivitäten verstanden werden als Aktivitäten des arbeitenden Menschen. Was die Tradition des politischen Denkens betrifft, so unterscheidet Arendt die frühe griechische Philosophie von der mit Platon und Aristoteles beginnenden, westlichen philosophischen Tradition. Das ist nicht unproblematisch, erlaubt ihr aber eine Verkürzung der Argumentation. Platon und Aristoteles begründen die abendländische philosophische Tradition, doch dieser Anfang bedeutet das Ende der Politik als Folge des Niedergangs der polis. Beide Denker sehen sich angesichts der Krise der unabhängigen Stadtstaaten konfrontiert mit der Frage, wie Menschen außerhalb der polis, gleichsam als Staatenlose leben können. 
We know today that Plato \& Arist.[toteles] were the end, rather than the beginning of greek philosophical thought which began its flight when Greece had reached or was near to reach its climax. But it remains true that Plato as well as Aristoteles became the beginners of occidental philosophical tradition and that this beginning, as distinguished from the beginning of Greek philos.[ophical] thought, was made in a time when everything political had come to an end. [...] In other words, we pay for truth with life. (S. 249)

Es erübrigt sich, darauf hinzuweisen, dass das Ende der griechischen Stadtstaaten nicht das Ende der Politik bewirkt hat; eher kann man von einer Globalisierung der Politik sprechen. Über den von Arendt angesprochenen Antagonismus zwischen Leben und Wahrheit möchte man Genaueres erfahren. Wieso das Problem der Arbeit das Politische, jenes der Geschichte die spirituelle Seite der ,perplexities which started at the end of the 18th [...] century" betrifft (S. 250), bleibt unbeantwortet. Immerhin erfährt man, dass wir aufgrund eben dieser Probleme Zeitgenossen von Marx bleiben. Das eigentlich revolutionäre, antitraditionale Element von Marx' Lehre sieht Arendt in der Glorifizierung der Arbeit und der Aufwertung der bislang von der Philosophie verachteten arbeitenden Klasse. Marx ist laut Arendt der einzige Denker, der die Emanzipation dieser Klasse als philosophisches Ereignis versteht. Vielleicht kann man nun der Argumentation einen Sinn abgewinnen. Nochmals wird der Umweg über Aristoteles gewählt: Die Freiheit der Herren erfordert die Unfreiheit der Sklaven. Sklaverei gehört nicht in den politischen Bereich. Gehen wir einen Schritt weiter: Freiheit gibt es laut Aristoteles und Arendt nur im politischen Bereich. Der von Marx erstrebten Befreiung der arbeitenden Klassen steht sein Wissen darum entgegen, dass Arbeit notwendig Zwang voraussetzt (MEW, Bd. 25, S. 828). So besteht denn seine "Sünde" wider die Tradition darin, dass die Emanzipation eben jenen Menschen politische Kompetenz zuschreibt, die unter naturbedingten Zwangsverhältnissen stehen. Doch Arendt verfehlt den Sinn von Marx' Argument. Um die bekannte Stelle zu zitieren: Das Reich der Freiheit beginnt dort, wo die Arbeit aufhört. In allen Gesellschaften sind Menschen gezwungen, zu arbeiten. Der Zwang kann durch die gemeinsame Kontrolle der Ökonomie gemindert werden, doch Arbeit bleibt ein Reich der Notwendigkeit. Jenseits dieses Reichs „beginnt die menschliche Kraftentwicklung, die sich als Selbstzweck gilt, das wahre Reich der Freiheit, das aber nur auf jenem Reich der Notwendigkeit als seiner Basis aufblühen kann“ (MEW, Bd. 25, S. 828).

Mit Marx habe erstmals in der Geschichte ein Denker, kein politischer Akteur, die Politik eines großen Landes inspiriert. Das kann man so sehen, übergeht dann aber eine ganze Reihe von bedeutenden früheren politisch einflussreichen Denkern. Marx sei gelungen, was Platon mit seinem sizilianischen Abenteuer versagt geblieben ist. Wer ihn verantwortlich mache für die totalitären Aspekte des Bolschewismus, sei sich nicht bewusst, dass entsprechende Anklagen sich gegen die gesamte westliche Tradition des politischen Denkens richteten, dessen letzter Vertreter Marx sei (S. 246). Ihm war klar, dass die politische Emanzipation der arbeitenden Klasse die Tradition des politischen Denkens erschüttern musste, dass diese die moderne Zeit der Revolutionen und der Industrialisierung nicht mehr begreifen konnte, da die politischen Kategorien dafür nicht gegeben waren. Die Bedeutung von Marx' 
Werk liegt im Eigensinn, mit dem er sich den Problemen der Arbeit und der Geschichte zuwendet. Er wusste im Gegensatz zu den utopischen Sozialisten, dass die Emanzipation nur in einer radikal veränderten Welt gelingen konnte. Ebenso hatte er entdeckt, dass die Arbeit selbst eine entscheidende Veränderung durchgemacht hatte. Sie wurde zur Quelle des Wohlstands und damit aller sozialen Werte; fortan waren alle Menschen ungeachtet ihrer Herkunft arbeitende Wesen. Marx lebte in einer sich radikal wandelnden Welt und seine Größe bestand in der Genauigkeit, mit der er das Zentrum des Wandels erfasste. Arendt weist bei dieser Gelegenheit auf den Unterschied von labour (Arbeit als lebenswichtige und nie endende Tätigkeit) und work (Produktion, Herstellung von Gegenständen, die von Dauer sind) hin, einen Unterschied der ihr zufolge von Marx verwischt wurde. Im „Kapital“ wird freilich sehr wohl auf den Unterschied zwischen labour und work hingewiesen (S. 251; vgl. MEW, Bd. 23, S. 61 f.).

Marx bleibt der philosophischen Tradition verpflichtet, wie Arendt mehrmals wiederholt. Allerdings wird bei ihm die arbeitende Klasse zur Erbin dieser Tradition. Ein Ende der Geschichte zeichnet sich ab, sobald die Revolution gelungen ist und die Klassenkonflikte überwunden sind. In jenem Augenblick wird das Ideal der griechischen Stadtstaaten erneut realisiert werden, doch werden die bislang verachtete Klasse der arbeitenden Menschen und deren Aktivitäten künftig die Philosophie beschäftigen müssen. Für Arendt stellt sich die Frage nach der Form der einer solchen Realität angemessenen Regierung. Sklaverei war in der westlichen Tradition nie eine Regierungsform und gehörte nicht zum Bereich der Politik. Marx hatte starken Einfluss auf die Politik, war aber, wie Arendt vermutet, an der Politik als solcher nicht sonderlich interessiert. Dies trifft auf den jungen Marx gewiss nicht zu, doch ist Arendt insofern zuzustimmen, als Marx unter dem Einfluss des damaligen Liberalismus die ältere Auffassung vom über der Gesellschaft stehenden Staat infrage stellte. Seine Glorifizierung der Arbeit bewirkte eine vollständige Umwälzung aller herkömmlichen politischen Werte. Arendt denkt dabei nicht an die politische Emanzipation der Arbeiterklasse, sondern daran, dass Arbeit nun ein erstrangiges politisches Faktum wurde. Arbeit als grundlegende und notwendige Aktivität war stets als ,Fluch“ empfunden worden, sie machte das Leben - fernab von den olympischen Göttern - beschwerlich. Das menschliche Leben sei der Notwendigkeit unterworfen und könne nie frei sein von Zwang. Menschen, die sich diesem Zwang unterwarfen, galten in „,unserer Tradition“ (,our tradition“, S. 253) als unfähig, die Aufgaben freier Bürger zu erfüllen. Arbeit ermöglicht es uns zu konsumieren; Arbeit als Metabolismus mit der Natur sei nicht produktiv, sondern konsumtiv. Da sie mit der biologischen Sphäre unseres Lebens verbunden sei, gehöre sie zur untersten, ,tierischen ' Funktion des Lebens und sei folglich eine rein private Angelegenheit eine bemerkenswerte Konklusion! Das politische Leben könne erst jenseits dieser Sphäre beginnen: Dort, wo die Bedürfnisse in eine gemeinsame Welt transzendiert werden könnten (S. 253 f). Erst die Herrschaft über Sklaven ermögliche eine Politik, die nicht auf Herrschaft und Unterwerfung beruhe.

Hier findet sich ein erster Ankerpunkt, der es erlaubt, Arendts Anliegen besser zu verstehen: Politik darf nicht mit Herrschaft gleichgesetzt werden, vielmehr handelt es sich um eine Aktivität zwischen Freien und Gleichen. Ein Zitat mag diesen Gedanken ausführen: „The rule over slaves for the Greeks was a pre-political 
condition of politics“. Anders formuliert: Sklaven müssen der Herrschaft unterworfen werden, doch handelt es sich dabei um eine private, nicht um eine öffentlichpolitische Handlungsweise. „This form of politics underwent a decisive change in the time of the decay of the Greek polis which coincided with the rise of Greek philosophy“ (S. 254). Die Geringschätzung der Philosophen bezog sich auf das politische Handeln, ein solches Handeln, das auf der Befreiung von den Zwängen des biologischen Lebens beruhte, wurde durch das philosophische Ideal ersetzt. Damit wurde die Sphäre der Politik kolonisiert von der Unterscheidung zwischen ,Herrschen und beherrscht werden'; die Herrschaft über die Notwendigkeiten des Lebens wurde zur Bedingung der Philosophie, nicht der Politik; alles, was den Menschen ein gutes Leben ermöglichte, wurde fortan als Politik bezeichnet (S. 254). Dies ist eine von vielen Stellen, bei denen es schwer fällt, der Logik von Arendt zu folgen. Ihre Darlegungen bleiben oft stichwortartig.

Was ist der Kern von Arendts kritischen Interpretationen von und Fragen an Marx’ Denken? Als Marx Arbeit zur wichtigsten Tätigkeit des Menschen erklärte, brachte er mit den Begriffen der Tradition zum Ausdruck, dass nicht die Freiheit, sondern der Zwang den Menschen zum Menschen mache. Wenn er dem beifügte, wer über andere herrsche, könne nicht frei sein, widersprach er sich, versprach er doch allen Menschen Freiheit und versagte sie zugleich allen. Er kehrte die eigentliche Bedeutung von Freiheit um: Freiheit vom Zwang, den wir natürlicher- und ursprünglicherweise in der condition humaine erleiden (S. 254). Die Emanzipation der arbeitenden Menschen war von solch revolutionärer Bedeutung, weil die westliche Haltung zur Arbeit so eng verbunden war mit ihrer Haltung zum Leben im rein biologischen Sinn. Wiederum eine bemerkenswerte Aussage. Es entsteht der Eindruck, Arendt wolle die elementarsten Einsichten der Anthropologie von den Füßen auf den Kopf stellen. Steckt der Widerspruch nicht vielleicht im Anspruch auf eine absolute Freiheit jenseits aller Not und allen Zwangs? Will Arendt die - zumindest vorderhand noch - unveränderbaren Zwänge, denen die menschliche Existenz ausgeliefert ist, leugnen? Die Sklaven machten sich der philopsychia, der Liebe des Lebens um des Lebens willen schuldig; der Held der Freien war Achilles, „a doer of deeds and a speaker of words", der ein kurzes Leben gegen den ,ewigen Ruhm' der Größe eintauschte (S. 198). Gewiss - doch hier befinden wir uns im Bereich des Mythos. Soll Politik mythisch gedeutet werden? Später dann waren es die Philosophen, die ihr Leben der Suche nach ,ewiger Wahrheit" oder der Rettung ihrer unsterblichen Seelen widmeten. Für all diese Menschen war Arbeit eine Tätigkeit, die jeder Würde ermangelte und selbst für politisches Handeln wertlos war. Was ist dann aber von Aristoteles' Aussage zu halten, der vollkommene Staat sei um des Lebens willen entstanden und bestehe um des vollkommenen Lebens willen? Marx hatte, so wird Arendt später in Erinnerung rufen, in Übereinstimmung mit der antiken Auffassung Notwendigkeit im Sinne der zwingenden Notdurft des Lebensprozesses verstanden und ,mehr als irgendjemand sonst dazu beigetragen, der politisch jedenfalls verderblichsten Lehre der Moderne" zu einem endgültigen Sieg zu verhelfen: dass „,das Leben der Güter höchstes und der Lebensprozess der Gesellschaft Zweck und Ende aller Politik sei“ “. ${ }^{3}$ Ebenso wird sie später festhalten,

\footnotetext{
${ }^{3}$ Dies.: Über die Revolution, Piper, München/Zürich 1985 (engl. 1963), S. 79.
} 
zwischen derjenigen Meinung, der Staat und das Politische seien eine für die Freiheit unentbehrliche Einrichtung, und jener, die in ihm eine für das Leben unentbehrliche Einrichtung sieht, liege ein unüberbrückbarer Gegensatz. ${ }^{4}$ Wie kann und soll der Mensch sich von seiner biologischen, ,tierischen' Sphäre trennen? Worum soll es in der politischen Sphäre gehen, wenn nicht um vitale Probleme?

Man wird in Arendts frühen Texten keine stringente Logik erwarten, allerdings sind die zahlreichen Wendungen nicht immer leicht nachzuvollziehen. Marx wird das Interesse an Politik einmal abgesprochen, andernorts ist von seiner Glorifizierung der Politik die Rede. Die westliche Tradition ist laut Arendt seit Platon und Aristoteles von der Verachtung für Politik bestimmt. Platon verzweifelte an der Politik, Aristoteles bezog politisches Handeln auf materielle Interessen. Marx' Denken ist voller Selbstwidersprüche - dies ist für Arendt bekanntlich nicht als Kritik zu verstehen, beweist doch der Widerspruch gerade die Qualität eines Denkens. Marx' Widersprüche erklären sich aus seiner Betonung der Freiheit (S. 274). Zu erwähnen sind auch die utopischen Elemente seines Denkens. Laut Arendt ist Marx' Utopie freier Selbsttätigkeit inspiriert von der Vorstellung der athenischen Polis im fünften vorchristlichen Jahrhundert. Marx meint nicht, Menschen sollten überhaupt nicht mehr arbeiten, vielmehr sollen sie selbstbestimmt arbeiten - Freiheit und Arbeit sind nicht zwingend unvereinbare Gegensätze, wie Arendt unbeirrt behauptet. Was Marx' Widersprüche betrifft, bemerkt Arendt, sie seien Ausdruck von drei historischen Bewegungen, den Revolutionen, der Industrialisierung und der Forderung nach Freiheit. Marx wäre somit eher der genaue Beobachter denn der Erzähler von Utopien. Nochmals sei Arendts Urteil über Marx zitiert: Es war seine Größe, die Arbeit in den Mittelpunkt seiner Lehre zu stellen, weil dies genau das war, wovon alle politische Philosophie, nachdem sie nicht mehr wagte, die Sklaverei zu rechtfertigen, die Augen abgewandt hatte (S. 93). Damit sei jedoch die politische Frage, welche die Notwendigkeit der Arbeit im menschlichen Leben und ihre alles beherrschende Rolle in der modernen Welt an uns stellt, noch nicht beantwortet. Marx' Glorifizierung der Arbeit habe keinen der Gründe beseitigt, welche die Tradition anzuführen wusste, wenn sie dem nur arbeitenden Menschen politische Gleichberechtigung und volle menschliche Freiheit aberkannte (S. 99). Doch wer ist „der nur arbeitende Mensch“? Was Arendt geflissentlich übersieht, ist der Umstand, dass Marx nicht die Arbeit abschaffen will, sondern die Selbstbestimmung des arbeitenden Menschen, seine Befreiung aus dem Status des Lohnarbeiters als Bedingung seiner Emanzipation versteht. Schließlich ist Marx ,the mouthpiece of the hidden forces of his time“ (S. 299). Ebenso ist er der Vollender der abendländischen Tradition politischen Denkens.

Our tradition of political philosophy, unhappily and fatefully, and from its very beginning has deprived political affairs, those activities which concern the common public realm which comes into being wherever men live together, of all dignity of there own. In the terms of Aristotle, politics is a means to an end, it has no end in and by itself. The proper end of politics is in a way its oppo-

\footnotetext{
4 Dies.: Was ist Politik. Fragmente aus dem Nachlaß, hrsg. v. Ursula Ludz, Piper, München/Zürich 1993,
} S. 70 . 
site, namely that non-participation in political affairs, that schole which is the condition of philosophy, or rather a life devoted to it. (S. 294)

So lautet die Konklusion von Arendts frühen philosophischen Reflexionen. Rätselhaft ist, weshalb überhaupt von politischer Philosophie die Rede ist. Folgenreich für die Geringschätzung der Politik ist der Umstand, dass Politik keinen eigenen Ursprung habe, sondern lediglich aufgrund des elementaren, vorpolitischen Faktums der biologischen Bedürfnisse entstanden sei, welches bewirke, dass die Menschen sich um des Überlebens willen gegenseitig brauchen (S. 295). Vielleicht am aussagekräftigsten ist folgendes Zitat:

The first to believe that the polis had come into existence because of the need of man to rule over the necessities of life and that politics consequently would be quite superfluous if man in his singularity were self-sufficient, was Plato and the first to elaborate this belief of Plato into a full-fledged theory was Aristotle, who started his book on politics with a discussion of rule over slaves because the ultimate goal of all politics was to him already the satisfactory management of material conditions. (S. 413)

Kurzum: Marx habe die Tradition des westlichen politischen Denkens vollendet, weil seit Platon und Aristoteles alle politischen Denker Materialisten gewesen seien - Philosophen, denen das gute Leben und dessen Voraussetzungen wichtiger waren als die Politik (S. 413). Nebenbei sei hier darauf verwiesen, dass Arendt (S. 434) Marx zu zitieren meint und nicht bemerkt, dass dieser Adam Smith zitiert.

Politische Philosophie betrifft die Haltung des Philosophen zur Politik; ihre Tradition beginnt mit der Abkehr des Philosophen von der Politik, der fortan seine Prinzipien den menschlichen Angelegenheiten auferlegt. Die Tradition kommt an ihr Ende, wenn ein Philosoph - hier Marx - die Philosophie überwindet, um sie in der Politik zu verwirklichen (S. 484). Marx' Identifikation von Handlung und Gewalt steht im Gegensatz zur Politik der griechischen Stadtstaaten. Nicht Gewalt wurde hier ausgeübt; was die Griechen (laut Aristoteles) von den gewalttätigen Barbaren unterschied, war ihre Fähigkeit des Sprechens. Es ist schon fast erschütternd zu sehen, wie sehr Arendt die athenische Geschichte idealisiert und verfälscht. Da sie die reale Politik in eine imaginierte höhere Welt reiner Freiheit verbannen will, kann sie von der realen Geschichte und Politik Athens völlig absehen. Diese Geschichte und Politik können mit einem Wort zusammengefasst werden: stasis, das heißt Aufstand, Rebellion, Dissens, Bürgerkrieg, Mord und Todschlag. Von Solon über Peisistratos, Kleisthenes, die Tyrannis der Dreißig, Ephialtes und schließlich Perikles lösten die Verfassungen sich ab. Ein steter Machtwechsel zwischen aristokratischen und demokratischen Parteien, der keineswegs stets gewaltlos vor sich ging, bestimmte Athens Geschichte. Es ist bezeichnend, dass Platon neben dem sophistes den stasiastikos, den Aufrührer und Parteimann, als größte Gefahr für die polis anführt. Anders gesagt: Er kann unter Politik stets nur Herrschaft verstehen (Platon: Politikos, 258e-259d), wie Arendt zu Recht vermerkt (S. 402). Das ,Gute und ,Wahre" wird von oben verkündet, jeder Disput ist unnütz, gefährlich, subversiv. Platon kann weder für Marx noch für Arendt als politischer Denker gelten. Erinnert sei hier auch an Aristoteles: „Denn gegenwärtig schwören sie in einigen Oligarchien 
so: ,und gegen das Volk werde ich übelgesinnt sein und zu seinem Schaden raten was ich kann“" (Aristoteles: Pol. V 9, 1310a 9-11). Als einzig authentischer Politiker bleibt Perikles übrig, gelobt von Marx (MEW, Bd. 2, S. 77) und als exemplarisch für den griechischen Politiker vorgestellt von Arendt (S. 507f). Unbestreitbar spielt Perikles für die frühe Geschichte der Demokratie eine dominierende Rolle, freilich sollten die von Thukydides überlieferten Stellen für das genommen werden, was sie waren: ein hochtrabendes Selbstlob, dessen Glanz auf ganz Athen abstrahlen sollte.

Was Marx betrifft, so scheint mir eine Stelle entscheidend zu sein. Marx wusste um die Unvereinbarkeit zwischen klassischem politischen Denken und den modernen Voraussetzungen der Politik, welche der bislang verachteten Arbeit eine neue Würde verschaffen und die altehrwürdige Freiheit unter Bedingungen universaler Gleichheit garantieren wollten. Er wusste, dass die neuen Ideale - Gleichheit, Menschenwürde, politische Rechte, Verteilungsgerechtigkeit - ungenügend waren. Die Unvereinbarkeit besteht zwischen dem Faktum, dass Arbeit ein Symbol der Unterwerfung bleibt, sowie deren Deutung als positiver Freiheit. Sein Irrtum bestand darin, die Philosophie retten zu wollen und sie zu diesem Zweck der Tyrannei der Notwendigkeit zu unterwerfen (S. 495).

Arendt bleibt ihrer idée fixe treu: Politik findet in einer jenseitigen Welt statt, fernab von realen Menschen mit ihren realen Sorgen. Sie malt in „Vita activa“ ein idyllisches Bild von Politik. Auf Mary McCarthys Frage, was im öffentlichen Raum überhaupt zu besprechen und zu verhandeln sei, bekannte sie freimütig, das wisse sie selbst auch nicht. ${ }^{5}$ Sie stellt fest, dass die selbstzweckhafte Politik als Ideal auch bei Aristoteles nicht zu finden ist. Handeln und Sprechen sind ihr zufolge dem Prinzip des l'art pour l'art verpflichtet, der Selbstdarstellung, dem Wettbewerb um Ruhm und Ehre, doch Ruhm und Ehre gewannen die Politiker in der Regel als Feldherrn, nicht im entspannten Plaudern.

Der eindrückliche Umfang des vorliegenden Bandes erklärt sich aus dem Umstand, dass viele Artikel und Entwürfe in mehreren Versionen abgedruckt sind. Wer alles lesen will, wird immer wieder auf Wiederholungen und geringfügige Abweichungen stoßen. Für ein wissenschaftlich interessiertes Publikum ist eine solche Edition zweifellos wünschenswert und nützlich. Hilfreich ist das sehr gründliche Nachwort, das zumindest ansatzweise Wege aus dem Dickicht der ineinander verflochtenen Entwürfe weist.

Eine Gesamtausgabe der Werke von Hannah Arendt ist, um es nochmals zu unterstreichen, seit Langem ein Desiderat. Zunächst einmal gilt dies für die wissenschaftliche Auseinandersetzung mit der Philosophie von Hannah Arendt, es gilt aber ebenso für ein weiteres interessiertes Publikum, das sich auf die unorthodoxe Philosophie- und Politikauffassung seinen Reim zu machen versucht. Arendt ist längst zum intellektuellen Star aufgestiegen, jedes wissenschaftlich noch so leichtgewichtige Werk - zu erinnern ist an den unglückseligen Einfall von dtv, „Die Freiheit, frei zu sein“ zu publizieren und zum philosophischen Bestseller zu küren - kann mit Aufmerksamkeit rechnen. Arendts Vorliebe für Provokation und Widerrede beschert ihr ein dankbares Publikum. Dass ihre Konzeption von Politik - Politik als Selbstzweck

\footnotetext{
5 Dies.: Ich will verstehen. Selbstauskünfte zu Leben und Werk, hrsg. v. Ursula Ludz, Piper, München/ Zürich 1996, S. 88 f.
} 
- unsinnig ist, ihre ,Erfindung ' von Kants politischer Philosophie problematisch und ihre Gegenüberstellung einer angeblich von Furcht und Not beherrschten Französischen mit einer davon freien Amerikanischen Revolution naiv, all dies ist bekannt. Gerade deshalb jedoch scheint mir eine Gesamtausgabe dringend nötig zu sein, sie wird neue Studien inspirieren, philosophische und politische Probleme präziser zu analysieren helfen. $\mathrm{Zu}$ hoffen ist, dass die kommenden Bände mit der gleichen editorischen und inhaltlichen Sorgfalt erstellt werden wie dies bei Band 3 und Band 6 der Fall ist. Negativ ins Gewicht fällt freilich der Zeitplan - wer wird sich bis 2031 gedulden können?

\section{Besprochene Literatur}

- Arendt, Hannah: Kritische Gesamtausgabe. Complete Works. Critical Edition, Bd. 3: Sechs Essays. Die verborgene Tradition, hrsg. v. Barbara Hahn unter Mitarbeit von Barbara Breysach/Christian Pischel, 503 S., Wallstein, Göttingen 2019.

- Arendt, Hannah: Kritische Gesamtausgabe. Complete Works. Critical Edition, Bd. 6: The Modern Challenge to Tradition: Fragmente eines Buchs, hrsg. v. Barbara Hahn/James McFarland unter Mitarbeit von Ingo Kieslich/Ingeborg Nordmann, 924 S., Wallstein, Göttingen 2018.

Prof. Dr. em. Urs Marti-Brander war von 1995 bis 2017 Professor für Politische Philosophie an der Universität Zürich in der Schweiz. Er hat zahlreiche Publikationen zu Demokratietheorie, globaler Politik, Menschenrechten, zu Friedrich Nietzsche, Michel Foucault, Hannah Arendt, Jean-Jacques Rousseau, Alexis de Tocqueville und Karl Marx veröffentlicht. 